\title{
Coupled Fixed Point Theorems for Contractive Type Mappings in Dislocated Metric Spaces
}

\author{
T.Senthil Kumar ${ }^{1 *}$ R.Jahir Hussain ${ }^{2}$ \\ 1P.G.Department of Mathematics, Arignar Anna Government Arts College, Musiri, \\ 621211 , Tamilnadu, India. \\ 2P.G.And Research Department of Mathematics, Jamal Mohamed College \\ (Autonomous), Tiruchirappalli-620 020, Tamil Nadu, India.
}

Abstract: The purpose of this paper is to prove some new coupled fixed point theorems for contractive type mappings defined on dislocated metric spaces. Also, we give an example to support our main theorem and some corollaries of the main result.

Keywords: coupled fixed point, dislocated metric spaces, Contractive type mappings

\section{Introduction and preliminaries}

In 2000, P. Hitzler and A. K. Seda [5] introduced the notion of dislocated metric space in which self distance of a point need not be equal to zero. They also generalized the famous Banach contraction principle in this space. The study of common fixed points of mappings in dislocated metric space satisfying certain contractive conditions has been at the center of vigorous research activity. Dislocated metric space plays very important role in topology, logical programming and in electronics engineering. C.T. Aage and J. N. Salunke [1], A. Isufati [6] established some important fixed point theorems in single and pair of mappings in dislocated metric space. K. Jha, D Panthi [7] established a common fixed point theorem in dislocated metric spaces. In 2006, Bhaskar et al introduced the notion of coupled fixed point and proved some fixed point results in this context.

Definition 1.1: Let $X$ be a nonempty set and let $d_{1}: X \times X \rightarrow[0,+\infty)$ be a function, called a dislocated metric (or simply $d_{l}$-metric) if the following conditions hold for any $\mathrm{x}, \mathrm{y}, \mathrm{z} \in \mathrm{X}$ :

(i)if $d_{l}(x, y)=0$, then $x=y$,

(ii) $\mathrm{d}_{1}(\mathrm{x}, \mathrm{y})=\mathrm{d}_{1}(\mathrm{y}, \mathrm{x})$,

(iii) $d_{1}(x, y) \leq d_{1}(x, z)+d_{l}(z, y)-d_{l}(z, z)$.

The pair $\left(\mathrm{X}, \mathrm{d}_{1}\right)$ is then called a dislocated metric space. It is clear that $\operatorname{ifd}_{1}(\mathrm{x}, \mathrm{y})=0$, then from (i), $x=y$. But if $x=y, \mathrm{~d}_{1}(\mathrm{x}, \mathrm{y})$ may not be 0 .

Example1.2: If $\mathrm{X}=[0,+\infty)$, then $\mathrm{d}_{\mathrm{l}}(\mathrm{x}, \mathrm{y})=\mathrm{x}+\mathrm{y}$ defines a dislocated metric $d_{l}$ on $X$.

Example1.3: If $X=[0,+\infty)$, then $d_{1}(x, y)=\max \{x, y\}$ defines a dislocated metric $d_{1}$ on $X$.

Definition 1.4: A sequence $\left\{x_{n}\right\}$ in a $d_{1}$-metric space $\left(X, d_{1}\right)$ is called a Cauchy sequence if given

$\varepsilon>0$, there exists $\mathrm{n}_{0} \in \mathrm{N}$ such that for all $\mathrm{n}, \mathrm{m} \geq \mathrm{n}_{0}$, we have $\mathrm{d}_{\mathrm{l}}\left(\mathrm{x}_{\mathrm{m}}, \mathrm{x}_{\mathrm{n}}\right)<\varepsilon$.

Definition 1.5: A sequence $\left\{x_{n}\right\}$ in a $d_{l}$-metric space converges with respect to $d_{l}$ if there exists $\mathrm{x} \in \mathrm{X}$ such that $\mathrm{d}_{\mathrm{l}}\left(\mathrm{x}_{\mathrm{n}}, \mathrm{x}\right) \rightarrow 0$ as $\mathrm{n} \rightarrow+\infty$. In this case, $x$ is called limit of $\left\{x_{n}\right\}$, and we write $\mathrm{x}_{\mathrm{n}} \rightarrow \mathrm{x}$.

Definition 1.6: A $d_{l}$-metric space $\left(X, d_{l}\right)$ is called complete if every Cauchy sequence in $X$ converges to a point in $X$.

Definition 1.7: An element $(x, y) \in X \times X$ is called a coupled fixed point of the mapping $F: X \times X \rightarrow$ $X$ if $F(x, y)=x$ and $F(y, x)=y$.

Example 1.8: Let $X=[0, \infty)$ and $F: X \times X \rightarrow X$ be defined by $F(x, y)=x+y$

For all $\mathrm{x}, \mathrm{y} \in \mathrm{X}$. It is easy to see that $\mathrm{F}$ has a unique coupled fixed point $(0,0)$.

\section{Main Results}

Theorem 2.1 Let (X, d) be a dislocated metric space. Suppose that the mapping

$\mathrm{F}: \mathrm{X} \times \mathrm{X} \rightarrow \mathrm{X}$ Satisfies

$$
\begin{aligned}
\mathrm{d}(\mathrm{F}(\mathrm{x}, \mathrm{y}), \mathrm{F}(\mathrm{u}, \mathrm{v})) & \leq \mathrm{ad}(\mathrm{x}, \mathrm{u})+\mathrm{bd}(\mathrm{y}, \mathrm{v}) \\
& +\mathrm{hd}(\mathrm{F}(\mathrm{x}, \mathrm{y}), \mathrm{u})+\mathrm{kd}(\mathrm{F}(\mathrm{u}, \mathrm{v}), \mathrm{x}) \\
& +\operatorname{ld}(\mathrm{F}(\mathrm{x}, \mathrm{y}), \mathrm{x})+\operatorname{md}(\mathrm{F}(\mathrm{u}, \mathrm{v}), \mathrm{u}) \\
& +\mathrm{qd}(\mathrm{F}(\mathrm{y}, \mathrm{x}), \mathrm{y})+\operatorname{rd}(\mathrm{F}(\mathrm{v}, \mathrm{u}), \mathrm{v}) \ldots(1)
\end{aligned}
$$

For all $\mathrm{x}, \mathrm{y}, \mathrm{u}, \mathrm{v} \in \mathrm{X}$, where $\mathrm{a}, \mathrm{b}, \mathrm{h}, \mathrm{k}, \mathrm{l}, \mathrm{m}, \mathrm{q}$ and $\mathrm{r}$ are non-negative constants with $\mathrm{a}+\mathrm{b}+\mathrm{h}+\mathrm{k}+\mathrm{l}+\mathrm{m}+\mathrm{q}+\mathrm{r}$ $<1 / 2$. Then $\mathrm{F}$ has a unique coupled fixed point.

Proof: Choose $\mathrm{x}_{0}, \mathrm{y}_{0} \in \mathrm{X}$ and set 


$$
\begin{gathered}
\mathrm{x}_{1}=\mathrm{F}\left(\mathrm{x}_{0}, \mathrm{y}_{0}\right), \quad \mathrm{y}_{1}=\mathrm{F}\left(\mathrm{y}_{0}, \mathrm{x}_{0}\right) \\
\quad \ldots \\
\mathrm{x}_{\mathrm{n}+1}=\mathrm{F}\left(\mathrm{x}_{\mathrm{n}}, \mathrm{y}_{\mathrm{n}}\right), \quad \mathrm{y}_{\mathrm{n}+1}=\mathrm{F}\left(\mathrm{y}_{\mathrm{n}}, \mathrm{x}_{\mathrm{n}}\right)
\end{gathered}
$$

From (2.1), we have

$$
\begin{aligned}
\mathrm{d}\left(\mathrm{x}_{\mathrm{n}}, \mathrm{x}_{\mathrm{n}+1}\right) & =\mathrm{d}\left(\mathrm{F}\left(\mathrm{x}_{\mathrm{n}-1}, \mathrm{y}_{\mathrm{n}-1}\right), \mathrm{F}\left(\mathrm{x}_{\mathrm{n}}, \mathrm{y}_{\mathrm{n}}\right)\right) \leq \operatorname{ad}\left(\mathrm{x}_{\mathrm{n}-1}, \mathrm{x}_{\mathrm{n}}\right)+\operatorname{bd}\left(\mathrm{y}_{\mathrm{n}-1}, \mathrm{y}_{\mathrm{n}}\right) \\
& +\operatorname{hd}\left(\mathrm{F}\left(\mathrm{x}_{\mathrm{n}-1}, \mathrm{y}_{\mathrm{n}-1}\right), \mathrm{x}_{\mathrm{n}}\right)+\operatorname{kd}\left(\mathrm{F}\left(\mathrm{x}_{\mathrm{n}}, \mathrm{y}_{\mathrm{n}}\right), \mathrm{x}_{\mathrm{n}-1}\right) \\
& +\operatorname{ld}\left(\mathrm{F}\left(\mathrm{x}_{\mathrm{n}-1}, \mathrm{y}_{\mathrm{n}-1}\right), \mathrm{x}_{\mathrm{n}-1}\right)+\operatorname{md}\left(\mathrm{F}\left(\mathrm{x}_{\mathrm{n}}, \mathrm{y}_{\mathrm{n}}\right), \mathrm{x}_{\mathrm{n}}\right) \\
& +\mathrm{qd}\left(\mathrm{F}\left(\mathrm{y}_{\mathrm{n}-1}, \mathrm{x}_{\mathrm{n}-1}\right), \mathrm{y}_{\mathrm{n}-1}\right)+\operatorname{rd}\left(\mathrm{F}\left(\mathrm{y}_{\mathrm{n}}, \mathrm{x}_{\mathrm{n}}\right), \mathrm{y}_{\mathrm{n}}\right)
\end{aligned}
$$

Similarly, we have

$$
\begin{aligned}
& \begin{aligned}
d\left(y_{n}, y_{n+1}\right) & =d\left(F\left(y_{n-1}, x_{n-1}\right), F\left(y_{n}, x_{n}\right)\right) \leq \operatorname{ad}\left(y_{n-1}, y_{n}\right)+\operatorname{bd}\left(x_{n-1}, x_{n}\right) \\
& +\operatorname{hd}\left(F\left(y_{n-1}, x_{n-1}\right), y_{n}\right)+\operatorname{kd}\left(F\left(y_{n}, x_{n}\right), y_{n-1}\right)+\operatorname{ld}\left(F\left(y_{n-1}, x_{n-1}\right), y_{n-1}\right) \\
& +\operatorname{md}\left(F\left(y_{n}, x_{n}\right), y_{n}\right)+q d\left(F\left(x_{n-1}, y_{n-1}\right), x_{n-1}\right)+\operatorname{rd}\left(F\left(x_{n}, y_{n}\right), x_{n}\right)
\end{aligned} \\
& \text { Therefore, by letting }
\end{aligned}
$$

$d_{n}=d\left(x_{n}, x_{n+1}\right)+d\left(y_{n}, y_{n+1}\right)$, we have

$d_{n}=d\left(x_{n}, x_{n+1}\right)+d\left(y_{n}, y_{n+1}\right) \leq \operatorname{ad}\left(x_{n-1}, x_{n}\right)+b d\left(y_{n-1}, y_{n}\right)$

$+\operatorname{hd}\left(\mathrm{F}\left(\mathrm{x}_{\mathrm{n}-1}, \mathrm{y}_{\mathrm{n}-1}\right), \mathrm{x}_{\mathrm{n}}\right)+\mathrm{kd}\left(\mathrm{F}\left(\mathrm{x}_{\mathrm{n}}, \mathrm{y}_{\mathrm{n}}\right), \mathrm{x}_{\mathrm{n}-1}\right)+\mathrm{d}\left(\mathrm{F}\left(\mathrm{x}_{\mathrm{n}-1}, \mathrm{y}_{\mathrm{n}-1}\right), \mathrm{x}_{\mathrm{n}-1}\right)$

$+\operatorname{md}\left(F\left(x_{n}, y_{n}\right), x_{n}\right)+q d\left(F\left(y_{n-1}, x_{n-1}\right), y_{n-1}\right)+\operatorname{rd}\left(F\left(y_{n}, x_{n}\right), y_{n}\right)$

$+\operatorname{ad}\left(\mathrm{y}_{\mathrm{n}-1}, \mathrm{y}_{\mathrm{n}}\right)+\mathrm{bd}\left(\mathrm{x}_{\mathrm{n}-1}, \mathrm{x}_{\mathrm{n}}\right)+\mathrm{d}\left(\mathrm{F}\left(\mathrm{y}_{\mathrm{n}-1}, \mathrm{x}_{\mathrm{n}-1}\right), \mathrm{y}_{\mathrm{n}}\right)+\mathrm{kd}\left(\mathrm{F}\left(\left(\mathrm{y}_{\mathrm{n}}, \mathrm{x}_{\mathrm{n}}\right), \mathrm{y}_{\mathrm{n}-1}\right)\right.$

$+\operatorname{ld}\left(F\left(y_{n-1}, x_{n-1}\right), y_{n-1}\right)+\operatorname{md}\left(F\left(y_{n}, x_{n}\right), y_{n}\right)+q d\left(F\left(\left(x_{n-1}, y_{n-1}\right), x_{n-1}\right)+\operatorname{rd}\left(F\left(x_{n}, y_{n}\right), x_{n}\right)\right.$

$\leq \operatorname{ad}\left(x_{n-1}, x_{n}\right)+b d\left(y_{n-1}, y_{n}\right)+h d\left(x_{n}, x_{n}\right)+\operatorname{kd}\left(x_{n+1}, x_{n-1}\right)+\operatorname{ld}\left(x_{n}, x_{n-1}\right)$

$+\operatorname{md}\left(x_{n+1}, x_{n}\right)+q d\left(y_{n}, y_{n-1}\right)+\operatorname{rd}\left(y_{n+1}, y_{n}\right)+\operatorname{ad}\left(y_{n-1}, y_{n}\right)+b d\left(x_{n-1}, x_{n}\right)$

$+h d\left(y_{n}, y_{n}\right)+\operatorname{kd}\left(y_{n+1}, y_{n-1}\right)+\operatorname{ld}\left(y_{n}, y_{n-1}\right)+\operatorname{md}\left(y_{n+1}, y_{n}\right)+q d\left(x_{n}, x_{n-1}\right)+\operatorname{rd}\left(x_{n+1}, x_{n}\right)$

$\leq \operatorname{ad}\left(x_{n-1}, x_{n}\right)+\operatorname{bd}\left(y_{n-1}, y_{n}\right)+h d\left(x_{n-1}, x_{n}\right)+h d\left(x_{n}, x_{n+1}\right)+\operatorname{kd}\left(x_{n}, x_{n-1}\right)+\operatorname{kd}\left(x_{n+1}, x_{n}\right)$

$+\operatorname{ld}\left(x_{n}, x_{n-1}\right)+\operatorname{md}\left(x_{n+1}, x_{n}\right)+q d\left(y_{n}, y_{n-1}\right)+\operatorname{rd}\left(y_{n+1}, y_{n}\right)+\operatorname{ad}\left(y_{n-1}, y_{n}\right)+b d\left(x_{n-1}, x_{n}\right)$

$+h d\left(y_{n-1}, y_{n}\right)+h d\left(y_{n}, y_{n+1}\right)+k d\left(y_{n}, y_{n-1}\right)+k d\left(y_{n+1}, y_{n}\right)+\operatorname{ld}\left(y_{n}, y_{n-1}\right)$

$+\operatorname{md}\left(\mathrm{y}_{\mathrm{n}+1}, \mathrm{y}_{\mathrm{n}}\right)+\mathrm{qd}\left(\mathrm{x}_{\mathrm{n}}, \mathrm{x}_{\mathrm{n}-1}\right)+\operatorname{rd}\left(\mathrm{x}_{\mathrm{n}+1}, \mathrm{x}_{\mathrm{n}}\right)$

$\leq\left(\begin{array}{c}a+b+h \\ +k+l+q\end{array}\right)\left[d\left(x_{n-1}, x_{n}\right)+d\left(y_{n-1}, y_{n}\right)\right]+\left(\begin{array}{c}h+k \\ +m+r\end{array}\right)\left[d\left(x_{n}, x_{n+1}\right)+d\left(y_{n}, y_{n+1}\right)\right]$

$d_{n} \leq \frac{(a+b+h+k+l+q)}{[1-(h+k+m+r)]} d_{n-1}, \quad d_{n} \leq \delta d_{n-1}$, where $\delta=\frac{(a+b+h+k+l+q)}{[1-(h+k+m+r)]}<1$

In general, we have for $\mathrm{n}=0,1,2 \ldots$

$\mathrm{d}_{\mathrm{n}} \leq \delta \mathrm{d}_{\mathrm{n}-1} \leq \delta^{2} \mathrm{~d}_{\mathrm{n}-2} \leq \cdots \leq \delta^{\mathrm{n}} \mathrm{d}_{0}$, Now, for all $\mathrm{m}>\mathrm{n}$,

$\mathrm{d}\left(\mathrm{x}_{\mathrm{m}}, \mathrm{x}_{\mathrm{n}}\right) \leq \mathrm{d}\left(\mathrm{x}_{\mathrm{n}}, \mathrm{x}_{\mathrm{n}+1}\right)+\mathrm{d}\left(\mathrm{x}_{\mathrm{n}+1}, \mathrm{x}_{\mathrm{n}+2}\right)+\cdots+\mathrm{d}\left(\mathrm{x}_{\mathrm{m}-1}, \mathrm{x}_{\mathrm{m}}\right)$ and

$d\left(y_{m}, y_{n}\right) \leq d\left(y_{n}, y_{n+1}\right)+d\left(y_{n+1}, y_{n+2}\right)+\cdots+d\left(y_{m-1}, y_{m}\right)$

Therefore, we have $\mathrm{d}\left(\mathrm{x}_{\mathrm{m}}, \mathrm{x}_{\mathrm{n}}\right)+\mathrm{d}\left(\mathrm{y}_{\mathrm{m}}, \mathrm{y}_{\mathrm{n}}\right) \leq \delta^{\mathrm{n}} \mathrm{d}_{0}+\delta^{\mathrm{n}-1} \mathrm{~d}_{0}+\cdots+\delta^{\mathrm{m}-1} \mathrm{~d}_{0}$

Thus we have $\left|\mathrm{d}\left(\mathrm{x}_{\mathrm{m}}, \mathrm{x}_{\mathrm{n}}\right)+\mathrm{d}\left(\mathrm{y}_{\mathrm{m}}, \mathrm{y}_{\mathrm{n}}\right)\right| \leq \frac{\delta^{\mathrm{n}}}{1-\delta}\left|\mathrm{d}_{0}\right|$

Which implies that $\left\{\mathrm{x}_{\mathrm{n}}\right\}$ and $\left\{\mathrm{y}_{\mathrm{n}}\right\}$ are Cauchy sequences in $\mathrm{X}$. By $\mathrm{X}$ is complete, there existx* $\mathrm{y}^{*} \in \mathrm{X}$, such that $\lim _{n \rightarrow \infty} x_{n}=x^{*}$ and $\lim _{n \rightarrow \infty} y_{n}=y^{*}$. We now show that $\left(x^{*}, y^{*}\right)$ is a coupled fixed point of $F$. Let $\epsilon>0$. Continuity of $\mathrm{F}$ at $\left(\mathrm{x}^{*}, \mathrm{y}^{*}\right)$ implies that, for given $\frac{\epsilon}{2}>0$, there exist $\mathrm{d}>0$, such that $d\left(\mathrm{x}^{*}, \mathrm{u}\right)+\mathrm{d}\left(\mathrm{y}^{*}, \mathrm{v}\right)<\mathrm{d}$ implies, $d\left(F\left(x^{*}, y^{*}\right), F(u, v)<\frac{\epsilon}{2}\right.$. Since $\left\{x_{n}\right\} \rightarrow x$ and $\left\{y_{n}\right\} \rightarrow y$, for $\phi=\min \left(\frac{\epsilon}{2}, \frac{d}{2}\right)>0$, there exist $n_{0}, m_{0}$, such that, for $n \geq n_{0}, \quad m \geq m_{0}$, we have $d\left(x_{n}, x^{*}\right)<\phi$ and $d\left(x_{m}, x^{*}\right)<\phi$. Therefore, for $n \in N, n \geq$ $\mathrm{x}\left\{\mathrm{n}_{0}, \mathrm{~m}_{0}\right\}$

$$
\begin{aligned}
\mathrm{d}\left(\mathrm{F}\left(\mathrm{x}^{*}, \mathrm{y}^{*}\right), \mathrm{x}^{*}\right) & \leq \mathrm{d}\left(\mathrm{F}\left(\mathrm{x}^{*}, \mathrm{y}^{*}\right), \mathrm{x}_{\mathrm{n}+1}\right)+\mathrm{d}\left(\mathrm{x}_{\mathrm{n}+1}, \mathrm{x}^{*}\right) \\
& =\mathrm{d}\left(\mathrm{F}\left(\mathrm{x}^{*}, \mathrm{y}^{*}\right), \mathrm{F}\left(\mathrm{x}_{\mathrm{n}}, \mathrm{y}_{\mathrm{n}}\right)\right)+\mathrm{d}\left(\mathrm{x}_{\mathrm{n}+1}, \mathrm{x}^{*}\right)<\frac{\epsilon}{2}+\phi \leq \epsilon,
\end{aligned}
$$

From which it follows that $\mathrm{F}\left(\mathrm{x}^{*}, \mathrm{y}^{*}\right)=\mathrm{x} *$. In a similar manner, we can show that $\mathrm{F}\left(\mathrm{y}^{*}, \mathrm{x}^{*}\right)=\mathrm{y} *$. Hence $\left(\mathrm{x}^{*}, \mathrm{y}^{*}\right)$ is a coupled fixed point of $\mathrm{F}$.

Corollary 2.1 Let $(X, d)$ be a dislocated metric space. Suppose that the mapping

$F: X \times X \rightarrow X \quad$ Satisfies $\quad d(F(x, y), F(u, v)) \leq a[d(x, u)+d(y, v)]+h[d(F(x, y), u)+d(F(u, v), x)]+$ $\mathrm{l}[\mathrm{d}(\mathrm{F}(\mathrm{x}, \mathrm{y}), \mathrm{x})+\mathrm{d}(\mathrm{F}(\mathrm{u}, \mathrm{v}), \mathrm{u})]+\mathrm{q}[\mathrm{d}(\mathrm{F}(\mathrm{y}, \mathrm{x}), \mathrm{y})+\mathrm{d}(\mathrm{F}(\mathrm{v}, \mathrm{u}), \mathrm{v})] \ldots$

For all $x, y, u, v \in X$, where $a, h, l$ and $q$ are non-negative constants with $a+h+1+q<1 / 2$.

Then $\mathrm{F}$ has a unique coupled fixed point.

Proof: We can prove this result by putting $\mathrm{a}=\mathrm{b}, \mathrm{h}=\mathrm{k}, \mathrm{l}=\mathrm{m}$ and $\mathrm{q}=\mathrm{r}$ in Theorem 2.1

Corollary 2.2: Let $(X, d)$ be a dislocated metric space. Suppose that the mapping $F: X \times X \rightarrow X$ satisfies $d(F(x, y), F(u, v)) \leq a\{[d(x, u)+d(y, v)]+[d(F(x, y), u)+d(F(u, v), x)]$

$$
+[\mathrm{d}(\mathrm{F}(\mathrm{x}, \mathrm{y}), \mathrm{x})+\mathrm{d}(\mathrm{F}(\mathrm{u}, \mathrm{v}), \mathrm{u})]+[\mathrm{d}(\mathrm{F}(\mathrm{y}, \mathrm{x}), \mathrm{y})+\mathrm{d}(\mathrm{F}(\mathrm{v}, \mathrm{u}), \mathrm{v})]\}
$$

For all $\mathrm{x}, \mathrm{y}, \mathrm{u}, \mathrm{v} \in \mathrm{X}$, where $\mathrm{a}$ is non-negative constant, with $\mathrm{a}<1 / 2$. Then $\mathrm{F}$ has a unique coupled fixed point. 
Proof: We can prove this result by putting $\mathrm{a}=\mathrm{b}=\mathrm{h}=\mathrm{k}=\mathrm{l}=\mathrm{m}=\mathrm{q}=\mathrm{r}$ in Theorem 2.1.

Corollary 2.3: Let $(X, d)$ be a dislocated metric space. Suppose that the mapping $F: X \times X \rightarrow X$ satisfies $\mathrm{d}(\mathrm{F}(\mathrm{x}, \mathrm{y}), \mathrm{F}(\mathrm{u}, \mathrm{v})) \leq \mathrm{hd}(\mathrm{F}(\mathrm{x}, \mathrm{y}), \mathrm{u})+\mathrm{kd}(\mathrm{F}(\mathrm{u}, \mathrm{v}), \mathrm{x})+\operatorname{ld}(\mathrm{F}(\mathrm{x}, \mathrm{y}), \mathrm{x})+\mathrm{md}(\mathrm{F}(\mathrm{u}, \mathrm{v}), \mathrm{u})$

$$
+\mathrm{qd}(\mathrm{F}(\mathrm{y}, \mathrm{x}), \mathrm{y})+\operatorname{rd}(\mathrm{F}(\mathrm{v}, \mathrm{u}), \mathrm{v})
$$

For all $\mathrm{x}, \mathrm{y}, \mathrm{u}, \mathrm{v} \in \mathrm{X}$, where $\mathrm{h}, \mathrm{k}, \mathrm{l}, \mathrm{m}, \mathrm{q}$, and $\mathrm{r}$ are non-negative constants

With $\mathrm{h}+\mathrm{k}+\mathrm{l}+\mathrm{m}+\mathrm{q}+\mathrm{r}<1 / 2$. Then $\mathrm{F}$ has a unique coupled fixed point.

Proof: We can prove this result by applying Theorem 2.1 by putting $a=b=0$.

Corollary 2.4: Let $(X, d)$ be a dislocated metric space. Suppose that the mapping $F: X \times X \rightarrow X$ Satisfies $d(F(x, y), F(u, v)) \leq a d(x, u)+b d(y, v)+h d(F(x, y), u)+k d(F(u, v), x)$

$$
+\operatorname{ld}(F(x, y), x)+\operatorname{md}(F(u, v), u)
$$

For all $\mathrm{x}, \mathrm{y}, \mathrm{u}, \mathrm{v} \in \mathrm{X}$, where $\mathrm{a}, \mathrm{b}, \mathrm{h}, \mathrm{k}, \mathrm{l}$, and $\mathrm{m}$ are non-negative constants

With $a+b+h+k+l+m<1 / 2$. Then $F$ has a unique coupled fixed point.

Proof: We can prove this result by putting $\mathrm{q}=\mathrm{r}=0$ in Theorem 2.1.

\section{Example}

Now, we introduce an example to support our results

\section{Example 3.1:}

On the set $X=[0,1]$, define $d: X \times X \rightarrow \mathbb{R}^{+}, d(x, y)=|x-y|$

Also, define $F: X \times X \rightarrow X, F(x, y)=\frac{(x+y)}{4}$

Then

(1) $\quad(X, d)$ is a complete dislocated metric space.

(2) For any $x, y, u, v \in X$.

$$
d(F(x, y), F(u, v)) \leq \frac{1}{4}[d(x, u)+d(y, v)]
$$

Proof:

Here we have $F(x, y)=\frac{(x+y)}{4}$

Therefore $\mathrm{d}(\mathrm{F}(\mathrm{x}, \mathrm{y}), \mathrm{F}(\mathrm{u}, \mathrm{v}))=\mathrm{d}\left(\frac{\mathrm{x}+\mathrm{y}}{4}, \frac{(\mathrm{u}+\mathrm{v})}{4}\right)=\frac{1}{4}[|(\mathrm{x}+\mathrm{y})-(\mathrm{u}+\mathrm{v})|]$

$$
\leq \frac{1}{4}\left[|(x-u)|+|(y-v)| \leq \frac{1}{4}[d(x, u)+d(y, v)]\right.
$$

Holds for all $\mathrm{x}, \mathrm{y} \in \mathrm{X}$. Here $(0,0)$ is the unique coupled fixed point of $\mathrm{F}$.

\section{References}

[1]. C.T. Aage and J.N. Salunke, Some results of fixed point theorem in Dislocated quasi-metric -spaces. Bulletin of Marathwada Mathematical Society, 9(2008), 1-5.

[2]. C.T. Aage and J.N. Salunke. The results on fixed points in dislocated and dislocated quasi-metric space. Appl. Math. Sci. 2(59): 2941-2948, 2008.

[3]. T. G. Bhaskar and V. Lakshmikantham, Fixed point theorems in partially ordered cone metric spaces and applications, Nonlinear Anal., 65 (2006), 825-832.

[4]. P. Hitzler. Generalized Metrics and Topology in Logic Programming semantics. Ph.d thesis, National University of Ireland, University College Cork, 2001

[5]. P. Hitzler and A. K. Seda, "Dislocated topologies," Journal of Electrical Engineering, vol. 51, no. 12, pp. 3-7, 2000.

[6]. A. Isufati. Fixed point theorems in dislocated quasi-metric space. Appl. Math. Sci. 4(5): 217-223, 2010

[7]. K. Jha and D. Panthi. A common fixed point theorem in dislocated metric spaces. Applied Mathematical Sciences, 6(91):4497:4503, 2012. 